\title{
A novel mutation in $B R C A 1$ linked to breast and ovarian cancer and a genotype-phenotype correlation
}

\author{
JOSEFA SALGADO ${ }^{1}$, JOSÉ M. ARAMENDÍA ${ }^{2}$, CRISTINA GUTIÉRREZ ${ }^{1}$, CARMEN GIL $^{1}$, \\ MAITANE ROBLES ${ }^{1}$ and JESÚS GARCÍA-FONCILLAS ${ }^{1,2}$ \\ ${ }^{1}$ Clinical Genetics Unit; ${ }^{2}$ Department of Oncology, University Clinic of Navarra (CUN), Pamplona 31008, Navarra, Spain
}

Received December 15, 2010; Accepted May 11, 2011

DOI: $10.3892 / \mathrm{ol} .2011 .328$

\begin{abstract}
We report a novel BRCAl germline 4156delAA mutation detected in a 41-year-old woman with breast and ovarian cancer. Genomic DNA was obtained from peripheral blood. Standard polymerase chain reactions and direct sequencing were performed. This mutation originates a premature stop at codon 1354 of BRCA1 protein and has not been documented in any published report to the best of our knowledge. The mutation was not observed in any other family studied. Since this novel mutation was associated with both breast and ovarian cancer, the genotype-phenotype correlation was investigated in a patient base of 30 families.
\end{abstract}

\section{Introduction}

Mutations in the breast and ovarian cancer susceptibility genes $B R C A 1$ and $B R C A 2$ are found in a high proportion of multiple-case families with breast cancer, particularly if one or more cases of patients with ovarian cancer are included. More than 400 distinct cancer-associated BRCA1 and BRCA2 mutations have been reported according to the Breast Cancer Information Core (BIC) database (http://www.nchgr.nih.gov/ bic), a widespread international reference for information regarding mutations and polymorphisms in the two genes. Effective screening for cancer-associated mutations in $B R C A I$ and $B R C A 2$ may aid in elucidating the molecular mechanisms of carcinogenesis, and is crucial to risk assessment and cancer treatment.

\section{Materials and methods}

A Spanish 41-year-old non-Ashkenazi female patient was diagnosed and treated for breast and ovarian cancer at another

Correspondence to: Dr Josefa Salgado, Clinical Genetics Unit, University Clinic of Navarra (CUN), Avda. Pio XII, 36, Pamplona 31008, Navarra, Spain

E-mail: jsalgadog@unav.es

Key words: novel BRCA1 germline mutation, breast and ovarian cancer center. Genomic DNA was obtained from peripheral blood and automatically extracted (MagNA Pure, Roche, Barcelona, Spain). Standard polymerase chain reactions were performed using AmpliTaq Gold polymerase from Perkin-Elmer (Waltham, MA, USA). Direct sequencing of the complete BRCAl was performed to the standard method on an automated sequencer ABI PRISM ${ }^{\circledR} 377$ Genetic Analyzer (Applied Biosystems, Carlsbad, CA, USA) and genetic variants were detected by comparison with a consensus wild-type sequence (GenBank NM_007294.2). Any mutation found was confirmed by repeated analysis, including reverse-primer sequencing of the suspicious exon. A group of 175 patients with a family history of ovarian and breast cancer were used to screen for the mutation found.

Finally, we analyzed the genotype-phenotype correlation in 30 families at our center. The subjects were eligible for inclusion into the study if they tested positive for a pathological $B R C A 1$ or $B R C A 2$ truncation mutation. Families with missense mutations that would not necessarily be expected to have the same phenotypic effect as would be produced by protein-truncating mutations at the same position in the gene were excluded.

In accordance with normal clinical practice all of the patients included in the study provided written informed consent prior to blood sample extraction and we followed the Good Clinical Practice guidelines previously approved by our Ethics Committee.

\section{Results and Discussion}

A 4156delAA frameshift mutation was found in exon 11 of $B R C A 1$, yet to be reported in the BIC database (Fig. 1A). Frequent truncations found in the Spanish population are 187delAG and 5385insC in BRCA1 and 3036del4, 3492insT, 5374del4, 9254delTCAT and 9538del2 in $\operatorname{BRCA2}(1,2)$. Geographical variations in the mutations distribution may be due to founder effects. The mutation in 175 families from various Spanish regions was analyzed with no positive results. Further investigations in low-represented Spanish areas may facilitate a description of genetic influences and associated phenotype features. The focus of this investigation was on two main areas: i) genotype-phenotype correlation and ii) the functional consequences of the BRCA1-truncated protein. Since the 4156delAA frameshift mutation is associ- 
A

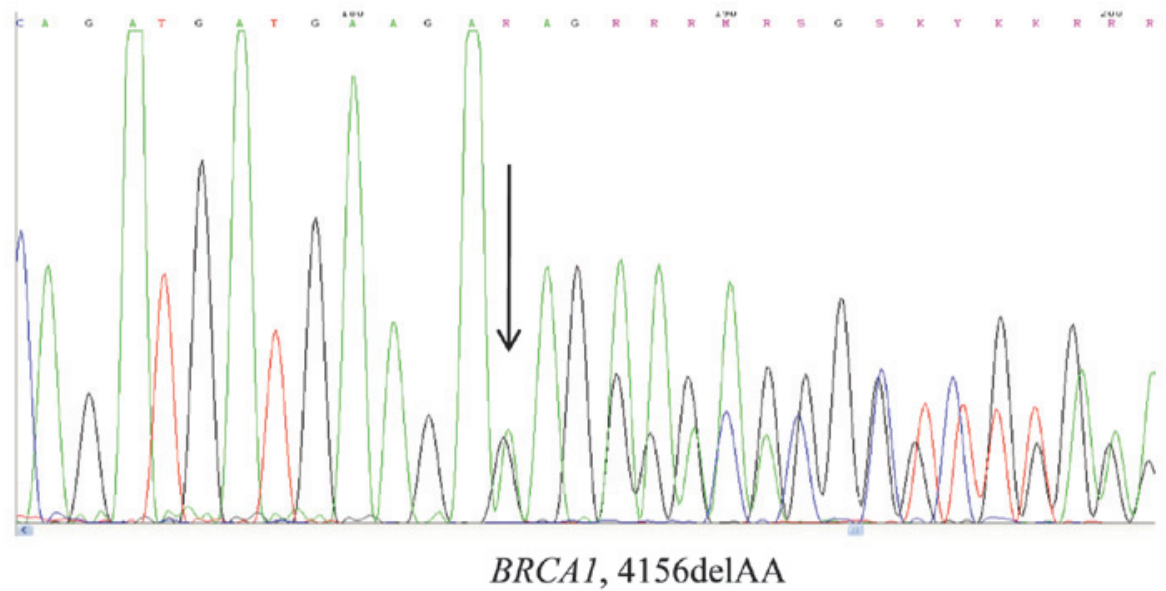

B BRCA1

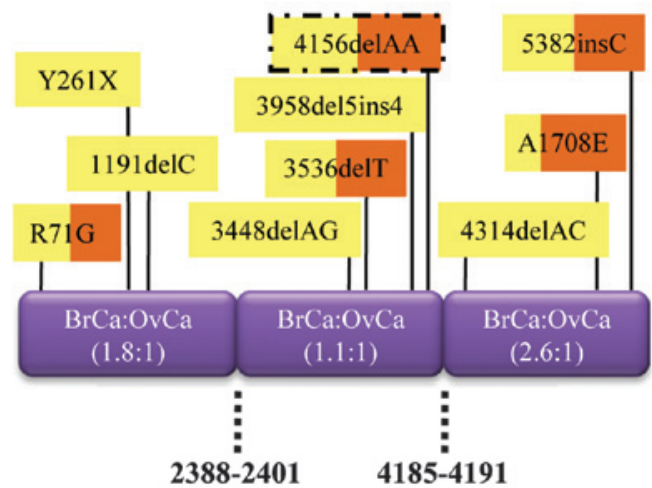

Breast cancer,
BRCA2

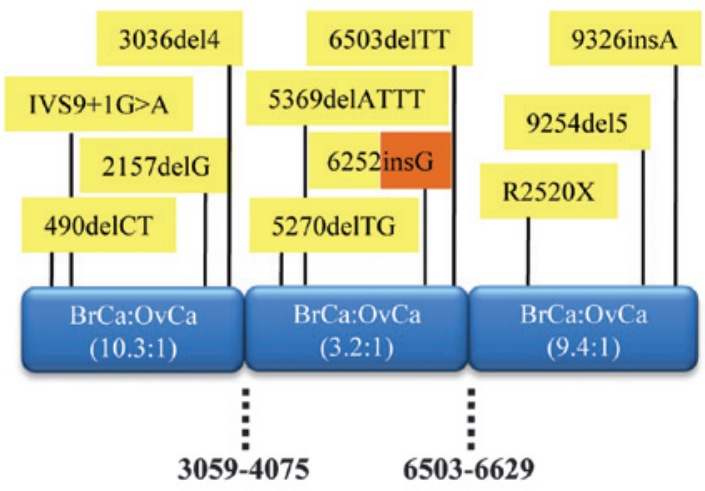

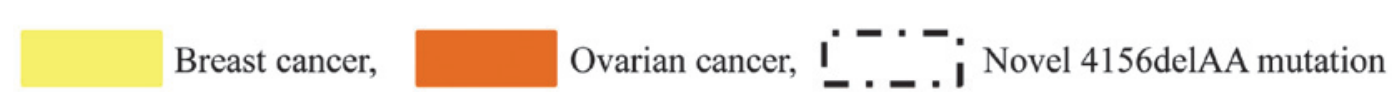

Figure 1. (A) Direct sequencing of the BRCA1 exon 11. Arrow indicates the 4156delAA mutation. (B) Location of germline mutations in 30 families, either in $B R C A 1$ or BRCA2. The BrC:OvC indicates the risk ratio of breast:ovarian cancers based on the studies of Thompson and Easton (5,6).

ated with breast and ovarian cancer in the same patient, we investigated the genotype-phenotype correlation in the mutations found in our sample.

In their study (3), Gayther et al studied groups of 32 and 25 families, providing evidence for a genotype-phenotype correlation in BRCA1 and BRCA2, respectively. These authors found that a linear trend model for the breast and ovarian cancer ratio was less satisfactory than a model dividing BRCA1/BRCA2 genes into discrete regions, and defined an ovarian cancer cluster region (OCCR) in BRCA2 $(3,4)$. Thompson and Easton (on behalf of the Breast Cancer Linkage Consortium) studied $B R C A 1$ and BRCA2 in 356 and 164 families, respectively, and proposed a division of $B R C A 1$ into three regions (of roughly equal size), with different associated risks showing an almost 1:1 ratio (breast and ovarian) in the center, and significantly higher risk for breast cancer in the $3^{\prime}$ and 5 ' regions $(5,6)$. Using the inclusion criteria indicated above, we studied the genotypephenotype correlation in a total of 30 families. The mutation spectrum constituted 16 frameshift deletions or insertions, two nonsense mutations, two missense mutations and one mutation affecting splicing [all mutations have been described in the BIC database or were recently published (7-9)]. The distribution of the mutations found and correlation with the phenotype are shown in Fig. 1B. Despite the limited number of families and mutations, our frequencies were in agreement with the expected frequencies proposed by the extensive study of Thompson and Easton $(5,6)$. The studies indicate that the novel 4156delAA mutation found in BRCAl is likely to exhibit a 1.1:1 associated risk for breast and ovarian cancers.

We have sought bibliographical information to further clarify the functional consequences of the BRCA1-truncated protein in order to gain a better understanding of biological mechanisms that may be responsible for the breast and ovarian cancer-risk variation among the different $B R C A 1$ and $B R C A 2$ gene regions. Cancer risk patterns are similar in the two genes, with the OCCR region in $B R C A 2$ and a central $B R C A 1$ region with a higher risk of ovarian cancer (Fig. 1B). Previously, the homologous recombination (HR) was found to be dependent on the interaction between $B R C A 1$ and BRCA2 (through the mediator protein PALB2), which is required for the recruitment of RAD51. The OCCR region coincides with the 8 BRC repeat motifs that modulate the DNA binding of RAD51. Additionally, BRCT domains of BRCA1 are required for the interaction and recruitment of BRCA2/RAD51 to the damage 
sites for execution of the HR-DNA repair function (10). In this context, the 4156delAA frameshift mutation detected originates a premature stop at codon 1354, leading to a truncated BRCA1 protein lacking the tandem BRCT C-terminal domains. Although it is possible that the positions of the risk-region boundaries relative to the RAD51-related domain are merely coincidental, it could be argued that the BRCA1 and BRCA2 proteins truncated midway in the RAD51-related domain behave differently from other truncated proteins outside this domain, leading to a higher ovarian cancer risk. On the other hand, another potential biological scenario is one in which the existence of in-frame alternative splicing of the BRCA proteins is assumed by skipping the RAD51-related domain, but retaining a certain degree of BRCA functionality (11). If these isoforms were more frequent in breast epithelial tissue than in ovarian epithelial tissue then the partial rescue and, thus, the reduced penetrance would be evident only in breast cancer. More detailed functional and population studies are required to clarify these hypotheses.

Finally, a 'BRCAness' syndrome in ovarian cancer has been associated with serous histology, longer treatment-free interval between relapses, improved overall survival and high response rates to first and subsequent lines of platinum-based treatment (12). BRCA1 is involved in the nucleotide excision repair of DNA adducts, since it has been reported that BRCA1 promotes the assembly of RAD51 after treatment with cisplatinum and that BRCA1-defective cells down-regulate ERCC1 $(13,14)$. Platinum-based compounds have not been included in conventional chemotherapy regimens for breast cancer. However, recent clinical studies, as well as potential molecular mechanisms, may indicate a rationale supporting the use of these compounds against hereditary and triple-negative phenotype breast cancer, particularly in the context of breast and ovarian cancer in the same patient.

In conclusion, the BRCAs genotype appears to have a significant impact on the molecular phenotype as well as on drug sensitivity. Although differences in risks are not currently sufficient to justify different clinical management according to the position of the mutation, it may prove useful for the provision of a more realistic assessment of the risk of breast and ovarian cancer in mutation carriers. Further large population-based studies of genotype-phenotype correlations and treatment outcome may improve the management of patients undergoing genetic testing. Therapeutic strategies benefiting from genetic scenarios provide a framework for individualized cancer treatments.

\section{Acknowledgements}

We are grateful to the patients and families who participated in our genetic counseling, and to the clinicians and genetic counselors of the Department of Oncology and the Clinical Genetics Unit for their support and encouragement. CG has an ADA fellowship from the University of Navarra, Navarra, Spain.

\section{References}

1. Vega A, Torres M, Martínez JI, Ruiz-Ponte C, Barros F and Carracedo A: Analysis of BRCA1 and BRCA2 in breast and breast/ovarian cancer families shows population substructure in the Iberian peninsula. Ann Hum Genet 66: 29-36, 2002.

2. Diez O, Osorio A, Duran M, et al: Analysis of BRCA1 and BRCA2 genes in Spanish breast/ovarian cancer patients: A high proportion of mutations unique to Spain and evidence of founder effects. Hum Mutat 22: 301-312, 2003.

3. Gayther SA, Warren W, Mazoyer S, et al: Germline mutations of the BRCA1 gene in breast and ovarian cancer families provide evidence for a genotype-phenotype correlation. Nat Genet 11: 428-433, 1995.

4. Gayther SA, Mangion J, Russell P, et al: Variation of risks of breast and ovarian cancer associated with different germline mutations of the BRCA2 gene. Nat Genet 15: 103-105, 1997.

5. Thompson D and Easton D (Breast Cancer Linkage Consortium): Variation in cancer risks, by mutation position, in BRCA2 mutation carriers. Am J Hum Genet 68: 410-419, 2001.

6. Thompson D and Easton D (Breast Cancer Linkage Consortium): Variation in BRCA1 cancer risks by mutation position. Cancer Epidemiol Biomarkers Prev 11: 329-336, 2002.

7. Salgado J, Gil C, Robles M and Garcia-Foncillas J: A novel BRCA1 mutation in a Spanish patient with ovarian cancer. Breast Cancer Res Treat 113: 71-73, 2009.

8. Salgado J, Aramendia JM, Gutierrez C, Gil C, Robles M and Garcia-Foncillas J: A novel BRCA2 mutation that segregates with breast and prostate cancer in a Spanish family. Breast Cancer Res Treat 121: 219-220, 2010.

9. Salgado J, Gutierrez C, Gil C, Robles M and Garcia-Foncillas J: Comparative disease pattern of a patient with a novel BRCA2 truncation and knockout models for BRCA2. Breast Cancer Res Treat 123: 291-293, 2010.

10. Wu X, Mondal G, Wang X, et al: Microcephalin regulates BRCA2 and Rad51-associated DNA double-strand break repair. Cancer Res 69: 5531-5536, 2009.

11. Orban TI and Olah E: Emerging roles of BRCA1 alternative splicing. Mol Pathol 56: 191-197, 2003.

12. Tan DS, Rothermundt C, Thomas K, et al: 'BRCAness' syndrome in ovarian cancer: A case-control study describing the clinical features and outcome of patients with epithelial ovarian cancer associated with BRCA1 and BRCA2 mutations. J Clin Oncol 26: 5530-5536, 2008

13. Bhattacharyya A, Ear US, Koller BH, Weichselbaum RR and Bishop DK: The breast cancer susceptibility gene BRCA1 is required for subnuclear assembly of Rad51 and survival following treatment with the DNA cross-linking agent cisplatin. J Biol Chem 275: 23899-23903, 2000.

14. Tassone P, Di Martino MT, Ventura M, et al: Loss of BRCA1 function increases the antitumor activity of cisplatin against human breast cancer xenografts in vivo. Cancer Biol Ther 8: 648-653, 2009 